\title{
Cost-effectiveness of robotics and artificial intelligence in healthcare
}

\author{
Focus on robot-assisted prostatectomy
}

\author{
Caroline Piccininni
}

\begin{abstract}
Using robot-assisted prostatectomy as an example, this article aims to demonstrate some benefits and drawbacks of implementing new technologies in healthcare from a cost-effectiveness perspective. Decision making regarding implementation is often complicated by the need to balance improved effectiveness and efficiency with high initial costs of acquiring and maintaining new technology. In the context of Canada's publicly funded healthcare system, it is particularly important to gather data relating to both short- and long-term outcomes and costs to inform such decision making before new technologies can be adapted for widespread use.
\end{abstract}

\section{POTENTIAL COST-EFFECTIVENESS OF NEW TECHNOLOGIES IN HEALTHCARE}

In the industry of artificial intelligence, healthcare is one of the fastest growing subsectors and is projected to reach $\$ 10$ billion in worldwide revenue within the next five years. ${ }^{1}$ This rapid growth results from the capacity of new technology to improve and, in some cases, replace many of the tasks that are currently undertaken by healthcare workers. Growth is further compounded by the increasing need for healthcare systems to manage rising costs, while maintaining or improving treatment outcomes.

There are many possibilities for the implementation of artificial intelligence in healthcare. Some examples include providing virtual assistance to patients, improving detection of disease, and assisting with clinical decisions. ${ }^{1-3} \mathrm{~A}$ multitude of new technology is constantly being developed and relatively little has yet to achieve widespread implementation. ${ }^{2}$ As such, most have not been studied extensively from a health economics point of view. With this being said, short-term cost savings and improved outcomes have been demonstrated for the usage of artificial intelligence in the setting of robot-assisted surgery, virtual nursing assistants, administrative workflow assistance, dosage error reduction, fraud detection, preliminary diagnosis, and automated imaging diagnosis. On the whole, it has been estimated that widespread application of artificial intelligence for healthcare purposes has the potential to improve outcomes by $30-40 \%$ and reduce costs by as much as $50 \%{ }^{2}$

There are several major barriers to the implementation of artificial intelligence technologies in healthcare. The most critical of these include: concerns about accuracy and safety, limited ability in the setting of new or exceptional clinical cases, lack of knowledge regarding artificial intelligence, resources required to train physicians, security, and regulation. In order for new technology to be of value in the healthcare setting, it must meet certain requirements, such as: having a positive impact on patient care and outcomes, increasing efficiency of medical practice, or reducing risks to healthcare staff. New technologies are often seen as inherently valuable since they are developed to purposefully meet one of these requirements. In the Canadian context, healthcare progress is dependent not only on the potential increased effectiveness, but also on the cost-effective use of resources and new technologies. Whether these technologies provide a high return on investment must be considered before implementation; important factors for consideration include both the initial cost and the projected operating cost. The return on investment can be high in some cases where there is a high initial cost but where operating costs are low. Likewise, the return on investment can be low when a seemingly attractive purchase price masks operating costs that ultimately undermine any future benefit. Another important consideration is the length of time that the new technology will remain cost-effective. Both the return on investment and service life can be challenging to determine, but are important factors to take into account in addition to short-term cost savings and improved outcomes.

\section{COST-EFFECTIVENESS OF ROBOT-ASSISTED UROLOGICAL SURGERY}

Robot-assisted radical prostatectomy for localised prostate cancer is an example of a procedure that's cost and effects have been studied quite extensively. ${ }^{5-12}$ Prostatectomies are also performed for non-cancerous conditions such as benign prostate hyperplasia. This surgical procedure involves the removal of the prostate, surrounding tissues, and seminal vesicles. Prostate cancer mainly affects men above 60 years of age with an age-standardized incidence rate of 110 per 100,000 in Canada. ${ }^{4}$ The gold standard treatment for localised prostate cancer is radical retropubic prostatectomy, in which the removal is performed through an 8 to $10 \mathrm{~cm}$ incision in the abdominal wall. Technological advancements allowed the introduction of laparoscopic prostatectomy, which is minimally invasive and performed through multiple small incisions. Use of the laparoscopic prostatectomy has been limited due to the advanced surgical skills required to execute the procedure. ${ }^{4}$ Robotassisted radical prostatectomy using the Da Vinci Surgical System, in which the surgical instruments are not in direct contact with the surgeon's hands, was introduced to urologic oncology in 2000. ${ }^{6}$ In the United States, an increasing number of men diagnosed with localised prostate cancer are choosing a surgical treatment option and robot-assisted surgery has become the favoured approach. ${ }^{3}$ 
Robot-assisted prostatectomy has been shown to be advantageous in terms of its early outcomes and perioperative morbidity and mortality. ${ }^{7}$ In a comparative effectiveness study conducted by the Canadian Agency for Drugs and Technology in Health, robot-assisted surgery was associated with statistically significant reductions in length of hospital stay, blood loss, and transfusion rates compared to open and laparoscopic prostatectomy. ${ }^{8}$ This study also showed that the robot-assisted procedure was associated with reduced positive margin rates in patients with tumours confined to the prostate. ${ }^{8}$ With positive margins, there comes an additional cost of radiotherapy. ${ }^{5}$ Both laparoscopic and robot-assisted prostatectomy are associated with the short-term outcomes including fewer surgical complications and deaths compared to the open procedure. ${ }^{9}$ In addition, both laparoscopic and robot-assisted prostatectomy have been demonstrated to provide improved continence and sexual function, which are two of the most substantial negative early outcomes associated with an open prostatectomy. ${ }^{10}$

Although these short-term outcomes appear promising, the statistical significance of the observed differences associated with robot-assisted prostatectomy does not necessarily imply clinical relevance. Furthermore, long-term outcomes have yet to be fully evaluated and will be critical in the assessment of robotic surgery in urology. Perhaps most importantly, neither the laparoscopic procedure nor the robot-assisted procedure have been shown to reduce the risk of death from prostate cancer or prevent cancer recurrence compared to the open procedure. ${ }^{11}$ Long-term cancer outcomes relating to the different surgical approaches have not been evaluated in a randomized controlled trial. In addition, improved postoperative outcomes over the open procedure, including rates of complications and pain, have not been consistently demonstrated beyond 12 weeks after either the laparoscopic or robot-assisted procedure. ${ }^{11}$ Evidence regarding time to return to work and longterm survival rates is also scarce and inconclusive. ${ }^{8}$ Both short- and long-term outcomes, especially functional outcomes and cancer control, are also strongly linked to surgeon skill and experience, another important factor to consider with the uptake of robotic surgical devices in medicine. ${ }^{5}$

In comparison to the laparoscopic and open procedures, cost is the main disadvantage of using the robotic alternative, including the large initial acquisition fee that exceeds \$2 million USD per unit. Other operating costs include: the cost per use that has been approximated at \$200 USD, the cost to maintain the device, and the cost to train surgeons to use the device. Considering current Canadian usage patterns and indications for robotic procedures, the net institutional cost for operating a robotics program for seven years is estimated to be $\$ 2.9$ million $\mathrm{CAD} .{ }^{8}$ Of note, prostatectomy was estimated to be the most expensive indication-specific program, with a net cost of $\$ 3.5$ million CAD over seven years. ${ }^{8}$ The incremental cost of the robot-assisted procedure is estimated at $\$ 3,860$ CAD per patient compared to open surgery and $\$ 4,625 \mathrm{CAD}$ per patient compared with laparoscopic surgery. ${ }^{8}$ It is important to note that this incremental cost can be lowered by increasing the annual caseload. ${ }^{12}$ Modelling by Ramsey et al demonstrated that excess cost can be reduced by maintaining a case volume for each device of 100-150 procedures per year. ${ }^{12}$ Other important disadvantages that have been cited are the lack of tactile feedback while using the robotic device and the increased operative time compared to open prostatectomy. ${ }^{13}$

\section{CONCLUSION}

New technologies have the potential to improve outcomes while producing long-term cost savings through a variety of applications in healthcare. Regarding the implementation of new technologies from a health economics perspective, more studies, especially those of long-term costs and outcomes, are required. Many such technologies, such as robotic surgical devices, have potential to increase efficiency and effectiveness but at the same time, are associated with large initial costs. Future data and analyses will demonstrate whether a balance between these can feasibly be achieved and assist healthcare providers in making informed decisions regarding the adoption of new technologies. This article has highlighted robot-assisted prostatectomy as one example of a medical procedure lacking evidence on long-term cost effectiveness but with proven short-term benefits, provided that institutions are able to maintain a sufficient caseload.

\section{REFERENCES}

1. Collier M, Fu R, Yin L, et al. Artificial intelligence: Healthcare's new nervous system [Internet]. Dublin, Ireland: Accenture; 2017 [cited 2018 Feb 2] Available from: https://www.accenture.com/ t20171215T032059Z_w_/us-en/_acnmedia/PDF-49/AccentureHealth-Artificial-Intelligence.pdf \#zoom $=50$.

2. Frost \& Sullivan. Cognitive computing and artificial intelligence systems in healthcare [Internet]. San Antonio, Texas: Frost \& Sullivan; 2015 [cited 2018 Feb 2] Available from: http://www.frost.com/sublib/ display-report.do?id=NFFE-01-00-00-00\&bdata=aHR0cHM6Ly93dz IuZnJvc3QuY29tL25ld3MvcHJlc3MtcmVsZWFzZXMvNjAwLWOtN iliaWxsaW9uLWFydGlmaWNpYWwtaW50ZWxsaWdlbmNlLXN5c3 RlbXMtcG9pc2VkLWRyYW1hdGljLW1hcmtldC1leHBhbnNpb24taG VhbHRoY2FyZUB\%2BQEJhY2tAfkAxNTMyNjA4Nzc4MzE3.

3. Barbash GI, Glied SA. New technology and health care costs: The case of robot-assisted surgery. N Engl J Med. 2010 Aug;363:701-704. https://doi.org/10.1056/NEJMp1006602

4. Canadian Cancer Statistics Advisory Committee. Canadian Cancer Statistics 2017 [Internet]. Toronto, ON: Canadian Cancer Society; 2017 [cited 2018 Feb 2] Available from: http://www.cancer.ca/CanadianCancer-Statistics-2017-EN.pdf.

5. Medical Advisory Secretariat. Robotic-assisted minimally invasive surgery for gynecologic and urologic oncology: an evidence-based analysis. Ont Health Technol Assess Ser [Internet]. 2010 Dec [cited 2018 Feb 2]; 10(27) 1-118. Available from: http://www.health.gov.on.ca/ english/providers/program/mas/tech/reviews/pdf/rev_robotic surgery_20101220.pdf.

6. da Vinci Surgery. da Vinci prostatectomy [Internet]. Sunnyvale, California: Intuitive Surgical, Inc.; 2018 [cited 2018 Feb 2] Available from http://www.davincisurgery.com/da-vinci-urology/da_vinci prostatectomy.php. 
7. Robertson C, Close A, Fraser C, et al. Relative effectiveness of robotassisted and standard laparoscopic prostatectomy as alternatives to open radical prostatectomy for treatment of localised prostate cancer: A systematic review and mixed treatment comparison meta-analysis. BJU International. 2013 Oct;112(6):798-812. https://doi.org/10.1111/ bju. 12247

8. Ho C, Tsakonas E, Tran K, et al. Robot-Assisted Surgery Compared with Open Surgery and Laparoscopic Surgery: Clinical Effectiveness and Economic Analyses [Internet]. Ottawa, ON: Canadian Agency for Drugs and Technologies in Health; 2011 Sept (CADTH Technology Report, No. 137) [cited 2018 Mar 9] Available from: http://www.cadth. ca/en/products/health-technology-assessment/publication/2682.

9. Yu H, Hevelone ND, Lipsitz SR, et al. Use, costs and comparative effectiveness of robotic assisted, laparoscopic and open urological surgery. J Urol. 2012 Apr;87(4):1392-1399. https://doi.org/10.1016/j. juro.2011.11.089

10. Ahmed K, Ibrahim A, Wang TT, et al. Assessing the cost-effectiveness of robotics in urological surgery: A systematic review. BJU International. 2012 Nov;110(10):1544-1556. https://doi.org/0.1111/ j.1464-410X.2012.11015.x

11. Ilic D, Evans SM, Allan CA, et al. Laparoscopic and robot-assisted versus open radical prostatectomy for the treatment of localized prostate cancer. Cochrane Database Syst Rev. 2017 Sept; Issue 9 (Art. No. CD009625). https://doi.org/10.1002/14651858.CD009625.pub2

12. Ramsay C, Pickard R, Robertson C, et al. Systematic review and economic modelling of the relative clinical benefit and costeffectiveness of laparoscopic surgery and robotic surgery for removal of the prostate in men with localised prostate cancer. Health Technol Assess. 2012 Feb;16(41):1-313. https://doi.org/10.3310/hta16410

13. Burgess SV, Atug F, Castle EP, et al. Cost analysis of radical retropubic, perineal, and robotic prostatectomy. J Endourol. 2006 Oct;20(10).

https://doi.org/10.1089/end.2006.20.827 